\title{
Erratum to "Manifolds with Bakry-Emery Ricci Curvature Bounded Below", Advances in Pure Mathematics, Vol. 6 (2016), 754-764
}

\author{
Issa Allassane Kaboye1, Bazanfaré Mahaman² \\ ${ }^{1}$ Faculté de Sciences et Techniques, Université de Zinder, Zinder, Niger \\ ${ }^{2}$ Département de Mathématiques et Informatique, Université Abdou Moumouni, Niamey, Niger \\ Email: allassanekaboye@yahoo.fr, bmahaman@yahoo.fr
}

Received: August 24, 2016

Accepted: October 14, 2016

Published: October 17, 2016

Copyright (c) 2017 by authors and Scientific Research Publishing Inc. This work is licensed under the Creative Commons Attribution International License (CC BY 4.0).

http://creativecommons.org/licenses/by/4.0/

\section{c) (i) Open Access}

The original online version of this article (Issa Allassane Kaboye, Bazanfaré Mahaman (2016) Manifolds with Bakry-Emery Ricci Curvature bounded below 6, 754-764. http://dx.doi.org/10.4236/apm.2016.611061 unfortunately contains a mistake. The author wishes to correct the errors.

Lemma 3.5. Let ( $\left.M, g, e^{-f} d v o l g\right)$ be a complete smooth metric measure space with Ric R $_{f} \geq 0$; fix $p \in M$; if there exists $c$ so that $|f(x)| \leq c$ then for $R \geq r>0$

$$
\frac{\operatorname{Vol}_{f}(B(p, R))}{\operatorname{Vol}_{f}(B(p, r))} \leq e^{4 c}\left(\frac{R}{r}\right)^{n}
$$

Proof

Let $x$ be a point in $M$ and let $\gamma:[0, r] \rightarrow M$ be a minimal geodesic joining $\mathrm{p}$ to $x$ and $\left(e_{i}(t)\right)_{1 \leq i \leq n-1}$ be a parallel orthonormal vector fields along $\gamma$. Set $Y_{i}(t)=\frac{t}{r} e_{i}(t)$.

By the second variation formula we have:

$$
\begin{aligned}
m(r) & =\Delta r \leq \sum_{i=1}^{n-1} I\left(Y_{i}, Y_{i}\right) \\
& =\int_{0}^{r}\left(\sum_{i=1}^{n-1}\left\|Y_{i}^{\prime}(t)\right\|^{2}-\left\langle R\left(Y_{i}(t), \gamma^{\prime}(t)\right) \gamma^{\prime}(t), Y_{i}(t)\right\rangle\right) \mathrm{d} t \\
& \leq \frac{1}{r^{2}} \int_{0}^{r}\left(n-1-t^{2} \operatorname{Ric}\left(\gamma^{\prime}(t)\right)\right) d t \\
& \frac{n-1}{r}+\int_{0}^{r} \frac{t^{2}}{r^{2}} \operatorname{Hess}(f)\left(\gamma^{\prime}, \gamma^{\prime}\right) \mathrm{d} t
\end{aligned}
$$




$$
\begin{aligned}
& \frac{n-1}{r}+\int_{0}^{r} \frac{t^{2}}{r^{2}}(f \circ \gamma)^{\prime \prime} \mathrm{d} t \\
& =\frac{n-1}{r}+\frac{1}{r^{2}} \int_{0}^{r} \frac{\mathrm{d}}{\mathrm{d} t}\left(t^{2}(f \circ \gamma)^{\prime}(t)\right) \mathrm{d} t-\frac{2}{r^{2}} \int_{0}^{r} t(f \circ \gamma)^{\prime}(t) \mathrm{d} t \\
& =\frac{n-1}{r}+\partial_{r} f-\frac{2}{r} f(x)+\frac{2}{r^{2}} \int_{0}^{r}(f \circ \gamma)(t) \mathrm{d} t
\end{aligned}
$$

Hence

$$
\begin{aligned}
m_{f}(r) & =\Delta r-\partial_{r} f=\frac{\partial}{\partial r}\left(\ln \left(A_{f}(r, \theta)\right)\right) \\
& \leq \frac{n-1}{r}+\partial_{r} f-\frac{2}{r} f(x)+\frac{2}{r^{2}} \int_{0}^{r}(f \circ \gamma)(t) \mathrm{d} t
\end{aligned}
$$

For all positive reals $r$ and $s$, integrating this relation we have:

$$
\begin{aligned}
\int_{r}^{s} m_{f}(t) \mathrm{d} t & =\ln \left(\frac{A_{f}(s, \theta)}{A_{f}(r, \theta)}\right) \leq\left(\frac{s}{r}\right)^{n-1}+2 \int_{r}^{s}\left(\frac{1}{t^{2}} \int_{0}^{t} f(u) \mathrm{d} u-\frac{1}{t} f(t)\right) \mathrm{d} t \\
& =\ln \left(\frac{s}{r}\right)^{n-1}-\left.\left(\frac{2}{t} \int_{0}^{t} f \mathrm{~d} t\right)\right|_{r} ^{s}+2 \int_{r}^{s} \frac{1}{t} f \mathrm{~d} t-2 \int_{r}^{s} \frac{1}{t} f \mathrm{~d} t \\
& =\ln \left(\frac{s}{r}\right)^{n-1}-\left.\left(\frac{2}{t} \int_{0}^{t} f \mathrm{~d} t\right)\right|_{r} ^{s} \leq \ln \left(\frac{s}{r}\right)^{n-1}+4 c
\end{aligned}
$$

Therefore we have $r^{n-1} A_{f}(s, \theta) \leq e^{4 c} A_{f}(r, \theta) s^{n-1}$. Hence

$$
\int_{0}^{R} \int_{S^{n-1}} r^{n-1} A_{f}(s, \theta) \mathrm{d} \theta \mathrm{d} r \leq e^{4 c} \int_{0}^{R} \int_{S^{n-1}} A_{f}(r, \theta) \mathrm{d} \theta \mathrm{d} r
$$

which implies

$$
\frac{R^{n}}{n} \int_{S^{n-1}} A_{f}(s, \theta) \mathrm{d} \theta \leq e^{4 c} s^{n-1} \int_{0}^{R} \int_{S^{n-1}} A_{f}(r, \theta) \mathrm{d} \theta \mathrm{d} r=e^{4 c} s^{n-1} \operatorname{vol}_{f}(B(p, R))
$$

and integrating from 0 to $R^{\prime}$ with respect to $s$ we obtain the conclusion. 